Research Article

\title{
Some Operator Inequalities on Chaotic Order and Monotonicity of Related Operator Function
}

\author{
Changsen Yang and Yanmin Liu \\ College of Mathematics and Information Science, Henan Normal University, Xinxiang 453002, China \\ Correspondence should be addressed to Changsen Yang; yangchangsen0991@sina.com
}

Received 24 March 2013; Accepted 24 April 2013

Academic Editor: Yisheng Song

Copyright (c) 2013 C. Yang and Y. Liu. This is an open access article distributed under the Creative Commons Attribution License, which permits unrestricted use, distribution, and reproduction in any medium, provided the original work is properly cited.

We will discuss some operator inequalities on chaotic order about several operators, which are generalization of Furuta inequality and show monotonicity of related Furuta type operator function.

\section{Introduction}

An operator $T$ is said to be positive (denoted by $T \geq 0$ ) if $(T x, x) \geq 0$ for all vectors $x$ in a Hilbert space, and $T$ is said to be strictly positive (denoted by $T>0$ ) if $T$ is positive and invertible.

Theorem LH (Löwner-Heinz inequality, denoted by (LH) briefly). If $A \geq B \geq 0$ holds, then $A^{\alpha} \geq B^{\alpha}$ for any $\alpha \in[0,1]$.

This was originally proved in $[1,2]$ and then in [3]. Although (LH) asserts that $A \geq B \geq 0$ ensures $A^{\alpha} \geq B^{\alpha}$ for any $\alpha \in[0,1]$, unfortunately $A^{\alpha} \geq B^{\alpha}$ does not always hold for $\alpha>1$. The following result has been obtained from this point of view.

Theorem $\mathbf{F}$ (Furuta inequality). If $A \geq B \geq 0$, then for each $r \geq 0$,

(i) $\left(B^{r / 2} A^{p} B^{r / 2}\right)^{1 / q} \geq\left(B^{r / 2} B^{p} B^{r / 2}\right)^{1 / q}$,

(ii) $\left(A^{r / 2} A^{p} A^{r / 2}\right)^{1 / q} \geq\left(A^{r / 2} B^{p} A^{r / 2}\right)^{1 / q}$

hold for $p \geq 0$ and $q \geq 1$ with $(1+r) q \geq p+r$.

The original proof of Theorem $\mathrm{F}$ is shown in [4], an elementary one-page proof is in [5], and alternative ones are in $[6,7]$. We remark that the domain of the parameters $p, q$, and $r$ in Theorem $\mathrm{F}$ is the best possible for the inequalities (i) and (ii) under the assumption $A \geq B \geq 0$; see [8].

We write $A \gg B$ if $\log A \geq \log B$ for $A, B>0$, which is called the chaotic order.
Theorem A. For A, B > 0, the following (i) and (ii) hold:

(i) $A \gg B$ holds if and only if $A^{r} \geq\left(A^{r / 2} B^{p} A^{r / 2}\right)^{r /(p+r)}$ for $p, r \geq 0$

(ii) $A \gg B$ holds if and only if for any fixed $\delta \geq 0$, $F_{A, B}(p, r)=A^{-r / 2}\left(A^{r / 2} B^{p} A^{r / 2}\right)^{(\delta+r) /(p+r)} A^{-r / 2}$ is a decreasing function of $p \geq \delta$ and $r \geq 0$.

(i) in Theorem A is shown in $[9,10]$, an excellent proof in [11], a proof in the case $p=r$ in [12], (ii) in $[9,10]$, and so forth.

Lemma B (see [11]). Let $A$ be a positive invertible operator, and let $B$ be an invertible operator. For any real number $\lambda$,

$$
\left(B A B^{*}\right)=B A^{1 / 2}\left(A^{1 / 2} B^{*} B A^{1 / 2}\right)^{\lambda-1} A^{1 / 2} B^{*} .
$$

Definition 1. Let $A_{n}, A_{n-1}, \ldots, A_{2}, A_{1}, B \geq 0, r_{1}, r_{2}, \ldots, r_{n} \geq$ 0 , and $p_{1}, p_{2}, \ldots, p_{n} \geq 0$ for a natural number $n$.

Let $C_{A_{i}, B}[n]$ be defined by

$$
\begin{gathered}
C_{A_{i}, B}[n] \\
=A_{n}^{r_{n} / 2}\left\{A _ { n - 1 } ^ { r _ { n - 1 } / 2 } \left[\cdots A_{3}^{r_{3} / 2}\left\{A_{2}^{r_{2} / 2}\left(A_{1}^{r_{1} / 2} B^{p_{1}} A_{1}^{r_{1} / 2}\right)^{p_{2}} A_{2}^{r_{2} / 2}\right\}^{p_{3}}\right.\right. \\
\left.\left.\times A_{3}^{r_{3} / 2} \cdots\right] A_{n-1}^{r_{n-1} / 2}\right\}^{p_{n}} A_{n}^{r_{n} / 2} .
\end{gathered}
$$


For example,

$$
\begin{gathered}
C_{A_{i}, B}[2]=A_{2}^{r_{2} / 2}\left(A_{1}^{r_{1} / 2} B^{p_{1}} A_{1}^{r_{1} / 2}\right)^{p_{2}} A_{2}^{r_{2} / 2}, \\
C_{A_{i}, B}[4]=A_{4}^{r_{4} / 2}\left\{A_{3}^{r_{3} / 2}\left[A_{2}^{r_{2} / 2}\left(A_{1}^{r_{1} / 2} B^{p_{1}} A_{1}^{r_{1} / 2}\right)^{p_{2}} A_{2}^{r_{2} / 2}\right]^{p_{3}}\right. \\
\left.\times A_{3}^{r_{3} / 2}\right\}^{p_{4}} A_{4}^{r_{4} / 2} .
\end{gathered}
$$

Let $q[n]$ be defined by

$$
q[n]=\left\{\cdots\left[\left(p_{1}+r_{1}\right) p_{2}+r_{2}\right] p_{3}+\cdots+r_{n-1}\right\} p_{n}+r_{n} .
$$

For example,

$$
\begin{gathered}
q[1]=p_{1}+r_{1}, \quad q[2]=\left(p_{1}+r_{1}\right) p_{2}+r_{2}, \\
q[4]=\left\{\left[\left(p_{1}+r_{1}\right) p_{2}+r_{2}\right] p_{3}+r_{3}\right\} p_{4}+r_{4} .
\end{gathered}
$$

For the sake of convenience, we define

$$
C_{A_{i}, B}[0]=B, \quad q[0]=1,
$$

and these definitions in (6) may be reasonable by (2) and (4).

Lemma 2. For $A_{n}, A_{n-1}, \ldots, A_{2}, A_{1}, B \geq 0$ and any natural number $n$, we have

(i) $C_{A_{i}, B}[n]=A_{n}^{r_{n} / 2} C_{A_{i}, B}[n-1]^{p_{n}} A_{n}^{r_{n} / 2}$,

(ii) $q[n]=q[n-1] p_{n}+r_{n}$.

Proof. (i) and (ii) can be easily obtained by definitions (2) and (4).

\section{Basic Results Associated with}

$$
C_{A_{i}, B}[n] \text { and } q[n]
$$

We will give some operator inequalities on chaotic order, and Theorem 5 is further extension of Theorem 3.1 in [13].

Lemma 3. If $A \gg B$, for $p \geq 0$ and $r \geq 0$, then $A \gg$ $\left(A^{r / 2} B^{p} A^{r / 2}\right)^{1 /(p+r)}$.

Proof. Since $A \gg B$, we can obtain the following inequality.

$A^{r} \geq\left(A^{r / 2} B^{p} A^{r / 2}\right)^{r /(p+r)}$ holds for $p \geq 0$ and $r \geq 0$ by (i) of Theorem A.

Take the logarithm on both sides of the previous inequality; that is,

$$
\log A^{r} \geq \log \left(A^{r / 2} B^{p} A^{r / 2}\right)^{r /(p+r)},
$$

therefor we have

$$
A \gg\left(A^{r / 2} B^{p} A^{r / 2}\right)^{1 /(p+r)} .
$$

Theorem 4. If $A_{n} \gg A_{n-1} \gg \cdots \gg A_{2} \gg A_{1} \gg B$ and $r_{1}, r_{2}, \ldots, r_{n} \geq 0, p_{1}, p_{2}, \ldots, p_{n} \geq 0$ for a natural number $n$. Then the following inequality holds:

$$
A_{n} \gg C_{A_{i}, B}[n]^{1 / q[n]},
$$

where $C_{A_{i}, B}[n]$ and $q[n]$ are defined in (2) and (4).

Proof. We will show (9) by mathematical induction. In the case $n=1$.

Since $A_{1} \gg B$ implies

$$
A_{1} \gg\left(A_{1}^{r_{1} / 2} B^{p_{1}} A_{1}^{r_{1} / 2}\right)^{r_{1} /\left(p_{1}+r_{1}\right)}
$$

holds for any $p_{1} \geq 0$ and $r_{1} \geq 0$ by Lemma 3 , whence (9) for $n=1$.

Assume that (9) holds for a natural number $k(1 \leq k<$ $n)$. We will show that (9) holds $r_{1}, r_{2}, \ldots, r_{k}, r_{k+1} \geq 0$ and $p_{1}, p_{2}, \ldots, p_{k}, p_{k+1} \geq 0$ for $k+1$.

Put $D=A_{k+1}, E=A_{k}$, and $F=C_{A_{i}, B}[k]^{1 / q[k]}$, and (9) holds for $n=k$ implying

$$
D \gg E \gg F>0 .
$$

Equation (11) yields the following by Lemma 3, for $r \geq 0$ and $p \geq 0$ :

$$
D \gg\left(D^{r / 2} F^{p} D^{r / 2}\right)^{1 /(p+r)},
$$

that is,

$$
A_{k+1} \gg\left(A_{k+1}^{r / 2} C_{A_{i}, B}[k]^{p / q[k]} A_{k+1}^{r / 2}\right)^{1 /(p+r)} .
$$

Put $r=r_{k+1}, p=q[k] p_{k+1}$ in (13), then by (ii) of Lemma 2, the exponential power $1 /(p+r)$ of the right hand side of (13) can be written as follows:

$$
\frac{1}{p+r}=\frac{1}{q[k] p_{k+1}+r_{k+1}}=\frac{1}{q[k+1]},
$$

and we have the following desired (15) by (12) and (13):

$$
\begin{aligned}
A_{k+1} & \gg\left\{A_{k+1}^{r_{k+1} / 2}\left(C_{A_{i}, B}[k]\right)^{p_{k+1}} A_{k+1}^{r_{k+1} / 2}\right\}^{1 / q[k+1]} \\
& =C_{A_{i}, B}[k+1]^{1 / q[k+1]}
\end{aligned}
$$

so that (15) shows that (9) holds for $k+1$. 
Theorem 5. If $A_{n} \gg A_{n-1} \gg \cdots \gg A_{2} \gg A_{1} \gg B$ and $r_{1}, r_{2}, \ldots, r_{n} \geq 0$ for a natural number $n$. For any fixed $\delta \geq 0$, let $p_{1}, p_{2}, \ldots, p_{n}$ be satisfied by

$$
\begin{aligned}
p_{1} & \geq \delta, \\
p_{2} & \geq \frac{\delta+r_{1}}{p_{1}+r_{1}}, \\
& \vdots \\
p_{k} & \geq \frac{\delta+r_{1}+r_{2}+\cdots+r_{k-1}}{q[k-1]}, \\
& \vdots \\
p_{n} & \geq \frac{\delta+r_{1}+r_{2}+\cdots+r_{n-1}}{q[n-1]} .
\end{aligned}
$$

The operator function $I_{k}\left(p_{k}, r_{k}\right)$ for any natural number $k$ such that $1 \leq k \leq n$ is defined by

$$
I_{k}\left(p_{k}, r_{k}\right)=A_{k}^{-r_{k} / 2} C_{A_{i}, B}[k]^{\left(\delta+r_{1}+r_{2}+\cdots+r_{k}\right) / q[k]} A_{k}^{-r_{k} / 2} .
$$

Then the following inequality holds:

$$
A_{k-1}^{r_{k-1} / 2} I_{k-1}\left(p_{k-1}, r_{k-1}\right) A_{k-1}^{r_{k-1} / 2} \geq I_{k}\left(p_{k}, r_{k}\right)
$$

for every natural number $k$ such that $1 \leq k \leq n$, where $C_{A_{i}, B}[n]$ and $q[n]$ are defined in (2) and (4).

Proof. Since $C_{A_{i}, B}[0]=B, q[0]=1$ in (6), we may define $I_{0}\left(p_{0}, r_{0}\right)=B^{\delta}$ for $p_{0}=r_{0}=0$.

Because $A_{1} \gg B$, then for any fixed $\delta \geq 0$,

$$
\begin{array}{r}
B^{\delta} \geq A_{1}^{-r_{1} / 2}\left(A_{1}^{r_{1} / 2} B^{p_{1}} A_{1}^{r_{1} / 2}\right)^{\left(\delta+r_{1}\right) /\left(p_{1}+r_{1}\right)} A_{1}^{-r_{1} / 2} \\
\text { for } p_{1} \geq \delta, r_{1} \geq 0,
\end{array}
$$

since $F_{A_{1}, B}\left(\delta, r_{0}\right) \geq F_{A_{1}, B}\left(p_{1}, r_{1}\right)$ holds by (ii) of Theorem A. And (19) can be expressed as

$$
B^{\delta}=A_{0}^{r_{0} / 2} I_{0}\left(p_{0}, r_{0}\right) A_{0}^{r_{0} / 2} \geq I_{1}\left(p_{1}, r_{1}\right) .
$$

We can apply Theorem 4, and we have the following (21) for any natural number $k$ such that $1 \leq k \leq n$ :

$$
A_{k+1} \gg A_{k} \gg C_{A_{i}, B}[k]^{1 / q[k]}
$$

Since $X \gg Y$ implies that $X^{t} \gg Y^{t}$ holds for any $t \geq 0$, (21) ensures

$$
A_{k+1}^{\delta+r_{1}+r_{2}+\cdots+r_{k}} \gg C_{A_{i}, B}[k]^{\left(\delta+r_{1}+r_{2}+\cdots+r_{k}\right) / q[k]} .
$$

Putting $A=A_{k+1}^{\delta+r_{1}+r_{2}+\cdots+r_{k}}, B_{1}=C_{A_{i}, B}[k]^{\left(\delta+r_{1}+r_{2}+\cdots+r_{k}\right) / q[k]}$ and applying (19) for $\delta=1$ and $A \gg B_{1}$, we have

$$
B_{1} \geq A^{-r / 2}\left(A^{r / 2} B_{1}^{p} A^{r / 2}\right)^{(1+r) /(p+r)} A^{-r / 2}
$$

holds for $p \geq 1$ and $r \geq 0$.
Putting $r_{k+1}=r\left(\delta+r_{1}+r_{2}+\cdots+r_{k}\right)$ in (23), then (23) can be rewritten by

$$
\begin{gathered}
B_{1} \geq A_{k+1}^{-r_{k+1} / 2}\left(A_{k+1}^{r_{k+1} / 2} C_{A_{i}, B}[k]^{\left(\left(\delta+r_{1}+r_{2}+\cdots+r_{k}\right) / q[k]\right) p}\right. \\
\left.\times A_{k+1}^{r_{k+1} / 2}\right)^{(1+r) /(p+r)} A_{k+1}^{-r_{k+1} / 2} .
\end{gathered}
$$

Putting $p=\left(q[k] p_{k+1}\right) /\left(\delta+r_{1}+r_{2}+\cdots+r_{k}\right) \geq 1$, since $p_{k+1} \geq\left(\delta+r_{1}+r_{2}+\cdots+r_{k}\right) / q[k]$ in (16), then we have

$$
\begin{aligned}
A_{k}^{r_{k} / 2} & I_{k}\left(p_{k}, r_{k}\right) A_{k}^{r_{k} / 2} \\
= & B_{1}=C_{A_{i}, B}[k]^{\left(\delta+r_{1}+r_{2}+\cdots+r_{k}\right) / q[k]} \\
\geq & A_{k+1}^{-r_{k+1} / 2} \\
& \times\left(A_{k+1}^{r_{k+1} / 2} C_{A_{i}, B}[k]^{\left(\left(\delta+r_{1}+r_{2}+\cdots+r_{k}\right) / q[k]\right) p} A_{k+1}^{r_{k+1} / 2}\right)^{(1+r) /(p+r)} \\
& \times A_{k+1}^{-r_{k+1} / 2} \\
= & A_{k+1}^{-r_{k+1} / 2} C_{A_{i}, B}[k+1]^{\left(\delta+r_{1}+r_{2}+\cdots+r_{k}+r_{k+1}\right) /(q[k+1])} A_{k+1}^{-r_{k+1} / 2} \\
= & I_{k+1}\left(p_{k+1}, r_{k+1}\right),
\end{aligned}
$$

and we have (18) for $k$ such that $1 \leq k \leq n$ by (25) and (20) since (20) means (18) for $k=1$.

Corollary 6. If $A_{n} \gg A_{n-1} \gg \cdots \gg A_{2} \gg A_{1} \gg B$ and $r_{1}, r_{2}, \ldots, r_{n} \geq 0$ for a natural number $n$. For any fixed $\delta \geq 0$, let $p_{1}, p_{2}, \ldots, p_{n}$ be satisfied by (16).

Then the following inequalities hold:

$$
\begin{aligned}
B^{\delta} \geq & A_{1}^{-r_{1} / 2}\left(A_{1}^{r_{1} / 2} B^{p_{1}} A_{1}^{r_{1} / 2}\right)^{\left(\delta+r_{1}\right) /\left(p_{1}+r_{1}\right)} A_{1}^{-r_{1} / 2} \\
\geq & A_{1}^{-r_{1} / 2} A_{2}^{-r_{2} / 2} \\
& \times\left[A_{2}^{r_{2} / 2}\left(A_{1}^{r_{1} / 2} B^{p_{1}} A_{1}^{r_{1} / 2}\right)^{p_{2}} A_{2}^{r_{2} / 2}\right]^{\left(\delta+r_{1}+r_{2}\right) /\left(\left(p_{1}+r_{1}\right) p_{2}+r_{2}\right)} \\
& \times A_{2}^{-r_{2} / 2} A_{1}^{-r_{1} / 2} \\
& \vdots \\
\geq & A_{1}^{-r_{1} / 2} A_{2}^{-r_{2} / 2} A_{3}^{-r_{3} / 3} \cdots A_{n-1}^{-r_{n-1} / 2} A_{n}^{-r_{n} / 2} \\
& \times C_{A_{i}, B}[n]^{\left(\delta+r_{1}+r_{2}+\cdots+r_{n}\right) / q[n]} \\
& \times A_{n}^{-r_{n} / 2} A_{n-1}^{-r_{n-1} / 2} \cdots A_{3}^{-r_{3} / 3} A_{2}^{-r_{2} / 2} A_{1}^{-r_{1} / 2}
\end{aligned}
$$

where $C_{A_{i}, B}[n], q[n]$, and $I_{k}\left(p_{k}, r_{k}\right)(1 \leq k \leq n)$ are defined in (2), (4), and (17). 
Proof. Applying (18) of Theorem 5 for $k$ such that $1 \leq k \leq n$, we have

$$
\begin{aligned}
B^{\delta}= & A^{r_{0} / 2} I_{0}\left(p_{0}, r_{0}\right) A^{r_{0} / 2} \\
\geq & I_{1}\left(p_{1}, r_{1}\right) \\
= & A_{1}^{-r_{1} / 2}\left(A_{1}^{r_{1} / 2} B^{p_{1}} A_{1}^{r_{1} / 2}\right)^{\left(\delta+r_{1}\right) /\left(p_{1}+r_{1}\right)} A_{1}^{-r_{1} / 2} \\
\geq & A_{1}^{-r_{1} / 2} I_{2}\left(p_{2}, r_{2}\right) A_{1}^{-r_{1} / 2} \\
= & A_{1}^{-r_{1} / 2} A_{2}^{-r_{2} / 2}\left[A_{2}^{r_{2} / 2}\left(A_{1}^{r_{1} / 2} B^{p_{1}} A_{1}^{r_{1} / 2}\right)^{p_{2}}\right. \\
& \left.\times A_{2}^{-r_{2} / 2} A_{1}^{-r_{1} / 2} A_{2}^{r_{2} / 2}\right]^{\left(\delta+r_{1}+r_{2}\right) /\left(\left(p_{1}+r_{1}\right) p_{2}+r_{2}\right)} \\
\vdots & \\
\geq & A_{1}^{-r_{1} / 2} A_{2}^{-r_{2} / 2} A_{3}^{-r_{3} / 3} \cdots A_{n-1}^{-r_{n-1} / 2} I_{n}\left(p_{n}, r_{n}\right) \\
& \times A_{n-1}^{-r_{n-1} / 2} \cdots A_{3}^{-r_{3} / 3} A_{2}^{-r_{2} / 2} A_{1}^{-r_{1} / 2} \\
= & A_{1}^{-r_{1} / 2} A_{2}^{-r_{2} / 2} A_{3}^{-r_{3} / 3} \cdots A_{n-1}^{-r_{n-1} / 2} A_{n}^{-r_{n} / 2} \\
& \times C_{A_{i}, B}[n]^{\left(\delta+r_{1}+r_{2}+\cdots+r_{n}\right) / q[n]} \\
& \times A_{n}^{-r_{n} / 2} A_{n-1}^{-r_{n-1} / 2} \cdots A_{3}^{-r_{3} / 3} A_{2}^{-r_{2} / 2} A_{1}^{-r_{1} / 2} .
\end{aligned}
$$

\section{Monotonicity Property on Operator Functions}

We would like to emphasize that the condition of Theorem 7 is stronger than Theorem 5, and moreover when we discuss monotonicity property on operator functions, we can only apply Theorem 7 .

Theorem 7. If $A_{n} \gg A_{n-1} \gg \cdots \gg A_{2} \gg A_{1} \gg B$ and $r_{1}, r_{2}, \ldots, r_{n} \geq 0, p_{1}, p_{2}, \ldots, p_{n} \geq 0$ for a natural number $n$. Then the following inequality holds:

$$
A_{n}^{r_{n}} \geq C_{A_{i}, B}[n]^{r_{n} / q[n]},
$$

where $C_{A_{i}, B}[n]$ and $q[n]$ are defined in (2) and (4).

Proof. We will show (28) by mathematical induction. In the case $n=1$.

Since $A_{1} \gg B$ implies

$$
A_{1} \geq\left(A_{1}^{r_{1} / 2} B^{p_{1}} A_{1}^{r_{1} / 2}\right)^{r_{1} /\left(p_{1}+r_{1}\right)}
$$

holds for any, $p_{1} \geq 0$ and $r_{1} \geq 0$ by (i) of Theorem A, whence (28) for $n=1$.

Assume that (28) holds for a natural number $k(1 \leq$ $k<n)$. We will show (28) for $r_{1}, r_{2}, \ldots, r_{k+1} \geq 0$ and $p_{1}, p_{2}, \ldots, p_{k}, p_{k+1} \geq 0$ for $k+1$.
We can obtain the following inequality from the hypothesis (28) for the case $n=k$ :

$$
A_{k}^{r_{k}} \geq C_{A_{i}, B}[k]^{r_{k} / q[k]},
$$

hence we have $A_{k+1} \gg A_{k} \gg C_{A_{i}, B}[k]^{1 / q[k]}$, and (i) of Theorem A ensures

$$
A_{k+1}^{r} \geq\left(A_{k+1}^{r / 2} C_{A_{i}, B}[k]^{p / q[k]} A_{k+1}^{r / 2}\right)^{r /(p+r)} \text { for } p, r \geq 0 .
$$

Putting $r=r_{k+1}$ and $p=q[k] p_{k+1}$, then we have the following inequality:

$$
\begin{aligned}
A_{k+1}^{r_{k+1}} & \geq\left(A_{k+1}^{r_{k+1} / 2} C_{A_{i}, B}[k]^{p_{k+1}} A_{k+1}^{r_{k+1} / 2}\right)^{r_{k+1} /\left(q[k] p_{k+1}+r_{k+1}\right)} \\
& =C_{A_{i}, B}[k+1]^{r_{k+1} / q[k+1]},
\end{aligned}
$$

so that (32) shows (28) for $k+1$.

Theorem 8. If $A_{n} \gg A_{n-1} \gg \cdots \gg A_{2} \gg A_{1} \gg B$ and $r_{1}, r_{2}, \ldots, r_{n} \geq 0$ for a natural number $n$. For any fixed $\delta \geq 0$, let $p_{1}, p_{2}, \ldots, p_{n}$ be satisfied by (16).

Then

$$
I_{n}\left(p_{n}, r_{n}\right)=A_{n}^{-r_{n} / 2} C_{A_{i}, B}[n]^{\left(\delta+r_{1}+r_{2}+\cdots+r_{n}\right) / q[n]} A_{n}^{-r_{n} / 2}
$$

is a decreasing function of both $r_{n} \geq 0$ and $p_{n}$ which satisfies

$$
p_{n} \geq \frac{\delta+r_{1}+r_{2}+\cdots+r_{n-1}}{q[n-1]},
$$

where $C_{A_{i}, B}[n]$ and $q[n]$ are defined in (2) and (4).

Proof. Since the condition (16) with $\delta \geq 0$ suffices (28) in Theorem 7 , we have the following inequality by Theorem 7; see (28).

We state the following important inequality (35) for the forthcoming discussion which is the inequality in (16):

$$
q[n]=q[n-1] p_{n}+r_{n} \geq \delta+r_{1}+r_{2}+\cdots+r_{n-1}+r_{n}
$$

because the inequality in (35) follows by (ii) of Lemma 2, and the inequality follows by

$$
q[n-1] p_{n} \geq \delta+r_{1}+r_{2}+\cdots+r_{n-1}
$$

obtained by (34).

(a) Proof of the result that $I_{n}\left(p_{n}, r_{n}\right)$ is a decreasing function of $p_{n}$.

Without loss of generality, we can assume that $p_{n}>0$. We can obtain the following inequality by (28) and by (i) of Lemma 2:

$$
\begin{aligned}
A_{n}^{r_{n}} \geq & C_{A_{i}, B}[n]^{r_{n} / q[n]}=\left(A_{n}^{r_{n} / 2} C_{A_{i}, B}[n-1]^{p_{n}} A_{n}^{r_{n} / 2}\right)^{r_{n} / q[n]} \\
= & A_{n}^{r_{n} / 2} C_{A_{i}, B}[n-1]^{p_{n} / 2} \\
& \times\left(C_{A_{i}, B}[n-1]^{p_{n} / 2} A_{n}^{r_{n}} C_{A_{i}, B}[n-1]^{p_{n} / 2}\right)^{\left(r_{n}-q[n]\right) / q[n]} \\
& \times C_{A_{i}, B}[n-1]^{p_{n} / 2} A_{n}^{r_{n} / 2},
\end{aligned}
$$


and (37) implies

$$
\begin{aligned}
& \left(C_{A_{i}, B}[n-1]^{p_{n} / 2} A_{n}^{r_{n}} C_{A_{i}, B}[n-1]^{p_{n} / 2}\right)^{\left(q[n]-r_{n}\right) / q[n]} \\
& \quad \geq C_{A_{i}, B}[n-1]^{p_{n}} .
\end{aligned}
$$

Put $\alpha=\omega / p_{n} \in[0,1]$ for $p_{n} \geq \omega \geq 0$, then we raise each side of (38) to the power $\alpha=\omega / p_{n} \in[0,1]$, then

$$
\begin{aligned}
& \left(C_{A_{i}, B}[n-1]^{p_{n} / 2} A_{n}^{r_{n}} C_{A_{i}, B}[n-1]^{p_{n} / 2}\right)^{\left(\left(q[n]-r_{n}\right) \omega\right) /\left(q[n] p_{n}\right)} \\
& \geq C_{A_{i}, B}[n-1]^{\omega} .
\end{aligned}
$$

Whence we have

$$
\begin{aligned}
& I_{n}\left(p_{n}, r_{n}\right) \\
& =A_{n}^{-r_{n} / 2}\left(A_{n}^{r_{n} / 2} C_{A_{i}, B}[n-1]^{p_{n}} A_{n}^{r_{n} / 2}\right)^{\left(\delta+r_{1}+r_{2}+\cdots+r_{n}\right) / q[n]} A_{n}^{-r_{n} / 2} \\
& =A_{n}^{-r_{n} / 2} \\
& \times\left\{\left(A_{n}^{r_{n} / 2} C_{A_{i}, B}[n-1]^{p_{n}}\right.\right. \\
& \left.\left.\times A_{n}^{r_{n} / 2}\right)^{(q[n]+q[n-1] \omega) / q[n]}\right\}^{\left(\delta+r_{1}+r_{2}+\cdots+r_{n}\right) /(q[n]+q[n-1] \omega)} \\
& \times A_{n}^{-r_{n} / 2} \\
& =A_{n}^{-r_{n} / 2}\left\{A_{n}^{r_{n} / 2} C_{A_{i}, B}[n-1]^{p_{n} / 2}\right. \\
& \times\left(C_{A_{i}, B}[n-1]^{p_{n} / 2} A_{n}^{r_{n}}\right. \\
& \left.\times C_{A_{i}, B}[n-1]^{p_{n} / 2}\right)^{(q[n-1] \omega) / q[n]} \\
& \left.\times C_{A_{i}, B}[n-1]^{p_{n} / 2} A_{n}^{r_{n} / 2}\right\}^{\left(\delta+r_{1}+r_{2}+\cdots+r_{n}\right) /(q[n]+q[n-1] \omega)} \\
& \times A_{n}^{-r_{n} / 2} \text { by Lemma } \mathrm{B} \\
& =A_{n}^{-r_{n} / 2}\left\{A_{n}^{r_{n} / 2} C_{A_{i}, B}[n-1]^{p_{n} / 2}\right. \\
& \times\left(C_{A_{i}, B}[n-1]^{p_{n} / 2} A_{n}^{r_{n}}\right. \\
& \left.\times C_{A_{i}, B}[n-1]^{p_{n} / 2}\right)^{\left(\left(q[n]-r_{n}\right) \omega\right) /\left(q[n] p_{n}\right)} \\
& \times C_{A_{i}, B}[n-1]^{p_{n} / 2} \\
& \left.\times A_{n}^{r_{n} / 2}\right\}^{\left(\delta+r_{1}+r_{2}+\cdots+r_{n}\right) /(q[n]+q[n-1] \omega)} A_{n}^{-r_{n} / 2} \\
& \geq A_{n}^{-r_{n} / 2}\left(A_{n}^{r_{n} / 2} C_{A_{i}, B}[n-1]^{p_{n} / 2} C_{A_{i}, B}[n-1]^{\omega}\right. \\
& \left.\times C_{A_{i}, B}[n-1]^{p_{n} / 2} A_{n}^{r_{n} / 2}\right)^{\left(\delta+r_{1}+r_{2}+\cdots+r_{n}\right) /\left(q[n-1]\left(p_{n}+\omega\right)+r_{n}\right)} \\
& \times A_{n}^{-r_{n} / 2} \\
& =I_{n}\left(p_{n}+\omega, r_{n}\right) \text {, }
\end{aligned}
$$

and the last inequality holds by LH because (39) and $\left(\delta+r_{1}+\right.$ $\left.r_{2}+\cdots+r_{n}\right) /\left(q[n-1]\left(p_{n}+\omega\right)+r_{n}\right) \in[0,1]$ which is ensured by (35) and $q[n]+q[n-1] \omega=q[n-1]\left(p_{n}+\omega\right)+r_{n} \geq q[n]$ by (4), so that $I_{n}\left(p_{n}, r_{n}\right)$ is a decreasing function of $p_{n}$.

(b) Proof of the result that $I_{n}\left(p_{n}, r_{n}\right)$ is a decreasing function of $r_{n}$.

Without loss of generality, we can assume that $r_{n}>0$. Raise each side of (28) to the power $\mu / r_{n} \in[0,1]$ for $r_{n} \geq \mu \geq$ 0 by $\mathrm{LH}$, then

$$
A_{n}^{\mu} \geq\left(A_{n}^{r_{n} / 2} C_{A_{i}, B}[n-1]^{p_{n}} A_{n}^{r_{n} / 2}\right)^{\mu / q[n]} .
$$

We state the following inequality by (ii) of Lemma 3 and (35):

$$
\begin{aligned}
q[ & n]-\left(\delta+r_{1}+r_{2}+\cdots+r_{n}\right) \\
& =q[n-1] p_{n}+r_{n}-\left(\delta+r_{1}+r_{2}+\cdots+r_{n}\right) \\
& =q[n-1] p_{n}-\left(\delta+r_{1}+r_{2}+\cdots+r_{n-1}\right) \geq 0 .
\end{aligned}
$$

Then we have

$$
\begin{aligned}
I_{n} & \left(p_{n}, r_{n}\right) \\
= & A_{n}^{-r_{n} / 2} C_{A_{i}, B}[n]^{\left(\delta+r_{1}+r_{2}+\cdots+r_{n}\right) / q[n]} A_{n}^{-r_{n} / 2} \\
= & A_{n}^{-r_{n} / 2}\left(A_{n}^{r_{n} / 2} C_{A_{i}, B}[n-1]^{p_{n}} A^{r_{n} / 2}\right)^{\left(\delta+r_{1}+r_{2}+\cdots+r_{n}\right) / q[n]} A_{n}^{-r_{n} / 2} \\
= & C_{A_{i}, B}[n-1]^{p_{n} / 2} \\
& \times\left(C_{A_{i}, B}[n-1]^{p_{n} / 2} A_{n}^{r_{n}}\right. \\
& \left.\times C_{A_{i}, B}[n-1]^{p_{n} / 2}\right)^{\left(\delta+r_{1}+r_{2}+\cdots+r_{n}-q[n]\right) / q[n]} C_{A_{i}, B}[n-1]^{p_{n} / 2} \\
= & C_{A_{i}, B}[n-1]^{p_{n} / 2} \\
& \times\left\{\left(C_{A_{i}, B}[n-1]^{p_{n} / 2} A_{n}^{r_{n}}\right.\right. \\
& \times C_{A_{i}, B}[n-1]^{p_{n} / 2} \\
& \left.\times C_{A_{i}, B}[n-1]^{p_{n} / 2}\right\}^{\left(\delta+r_{1}+r_{2}+\cdots+r_{n}-q[n]\right) /(q[n]+\mu)} \\
& \left.\left.\times C_{A_{i}, B}[n-1]^{p_{n} / 2}\right)^{(q[n]+\mu) / q[n]}\right\}^{\left(\delta+r_{1}+r_{2}+\cdots+r_{n}-q[n]\right) /(q[n]+\mu)} \\
= & C_{A_{i}, B}[n-1]^{p_{n} / 2} \\
& \times\left\{C_{A_{i}, B}[n-1]^{p_{n} / 2} A_{n}^{r_{n} / 2}\right. \\
& \times\left(A_{n}^{r_{n} / 2} C_{A_{i}, B}[n-1]^{p_{n}} A_{n}^{r_{n} / 2}\right)^{\mu / q[n]} A_{n}^{r_{n} / 2} \\
& \\
& \\
& \\
& \\
&
\end{aligned}
$$




$$
\begin{aligned}
\geq & C_{A_{i}, B}[n-1]^{p_{n} / 2} \\
& \times\left\{C_{A_{i}, B}[n-1]^{p_{n} / 2} A_{n}^{r_{n}+\mu}\right. \\
& \left.\times C_{A_{i}, B}[n-1]^{p_{n} / 2}\right\}^{\left(\delta+r_{1}+r_{2}+\cdots+r_{n}-q[n]\right) /(q[n]+\mu)} \\
& \times C_{A_{i}, B}[n-1]^{p_{n} / 2} \\
= & I_{n}\left(p_{n}, r_{n}+\mu\right)
\end{aligned}
$$

and the last inequality holds by LH because (41) and

$$
\begin{aligned}
\frac{\delta+r_{1}+r_{2}+\cdots+r_{n}-q[n]}{q[n]+\mu} \\
=-\frac{q[n]-\left(\delta+r_{1}+r_{2}+\cdots+r_{n}\right)}{q[n]+\mu} \in[-1,0],
\end{aligned}
$$

so that $I_{k}\left(p_{k}, r_{k}\right)$ is a decreasing function of $r_{n}$.

\section{Acknowledgments}

This work was supported by the National Natural Science Foundation of China (1127112; 11201127), Technology and Pioneering project in Henan Province (122300410110).

\section{References}

[1] E. Heinz, "Beiträge zur Störungstheorie der Spektralzerlegung," Mathematische Annalen, vol. 123, pp. 415-438, 1951.

[2] K. Löwner, “Über monotone Matrixfunktionen," Mathematische Zeitschrift, vol. 38, no. 1, pp. 177-216, 1934.

[3] G. K. Pedersen, "Some operator monotone functions," Proceedings of the American Mathematical Society, vol. 36, pp. 309-310, 1972.

[4] T. Furuta, " $A \geq B$ assures $\left(B^{r} A^{p} B^{r}\right)^{1 / q} \geq B^{p+r / q}$ for $r \geq 0, p \geq$ $0, q \geq 1$ with $(1+2 r) q \geq p+2 r$," Proceedings of the American Mathematical Society, vol. 101, no. 1, pp. 85-88, 1987.

[5] T. Furuta, "An elementary proof of an order preserving inequality," Proceedings of the Japan Academy, vol. 65, no. 5, p. 126, 1989.

[6] M. Fujii, "Furuta's inequality and its mean theoretic approach," Journal of Operator Theory, vol. 23, no. 1, pp. 67-72, 1990.

[7] E. Kamei, "A satellite to Furuta's inequality," Mathematica Japonica, vol. 33, no. 6, pp. 883-886, 1988.

[8] K. Tanahashi, "Best possibility of the Furuta inequality," Proceedings of the American Mathematical Society, vol. 124, no. 1, pp. 141-146, 1996.

[9] T. Furuta, "Applications of order preserving operator inequality," Operator Theory, vol. 59, pp. 180-190, 1992.

[10] M. Uchiyama, "Some exponential operator inequalities," Mathematical Inequalities and Applications, vol. 2, no. 3, pp. 469-471, 1999.

[11] T. Furuta, "Extension of the Furuta inequality and Ando-Hiai log-majorization," Linear Algebra and Its Applications, vol. 219, pp. 139-155, 1996.

[12] M. Fujii, T. Furuta, and E. Kamei, "Furuta's inequality and its application to Ando's theorem," Linear Algebra and Its Applications, vol. 179, pp. 161-169, 1993.
[13] T. Furuta, "Operator functions on chaotic order involving order preserving operator inequalities," Journal of Mathematical Inequalities, vol. 6, no. 1, pp. 15-31, 2012. 


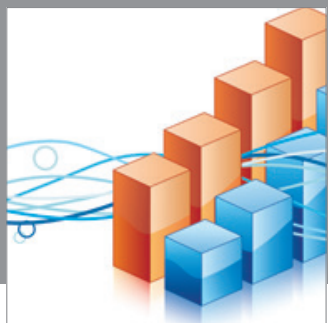

Advances in

Operations Research

mansans

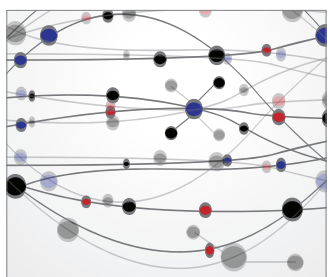

The Scientific World Journal
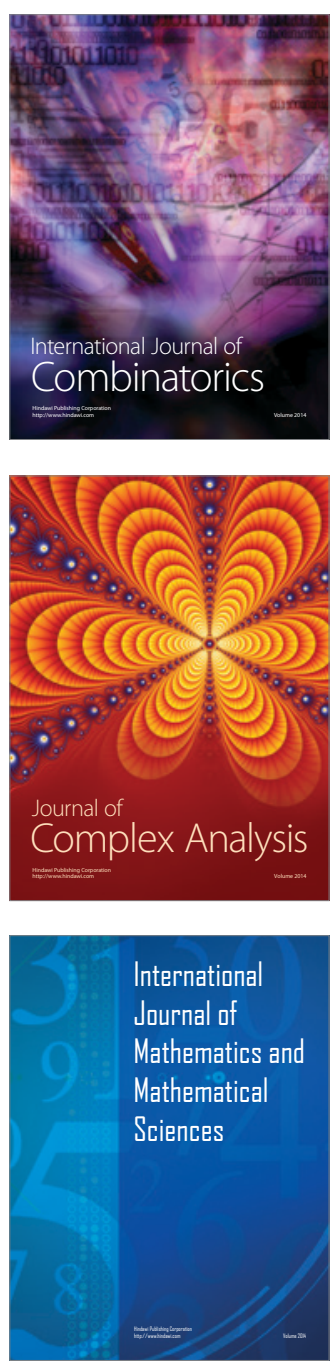
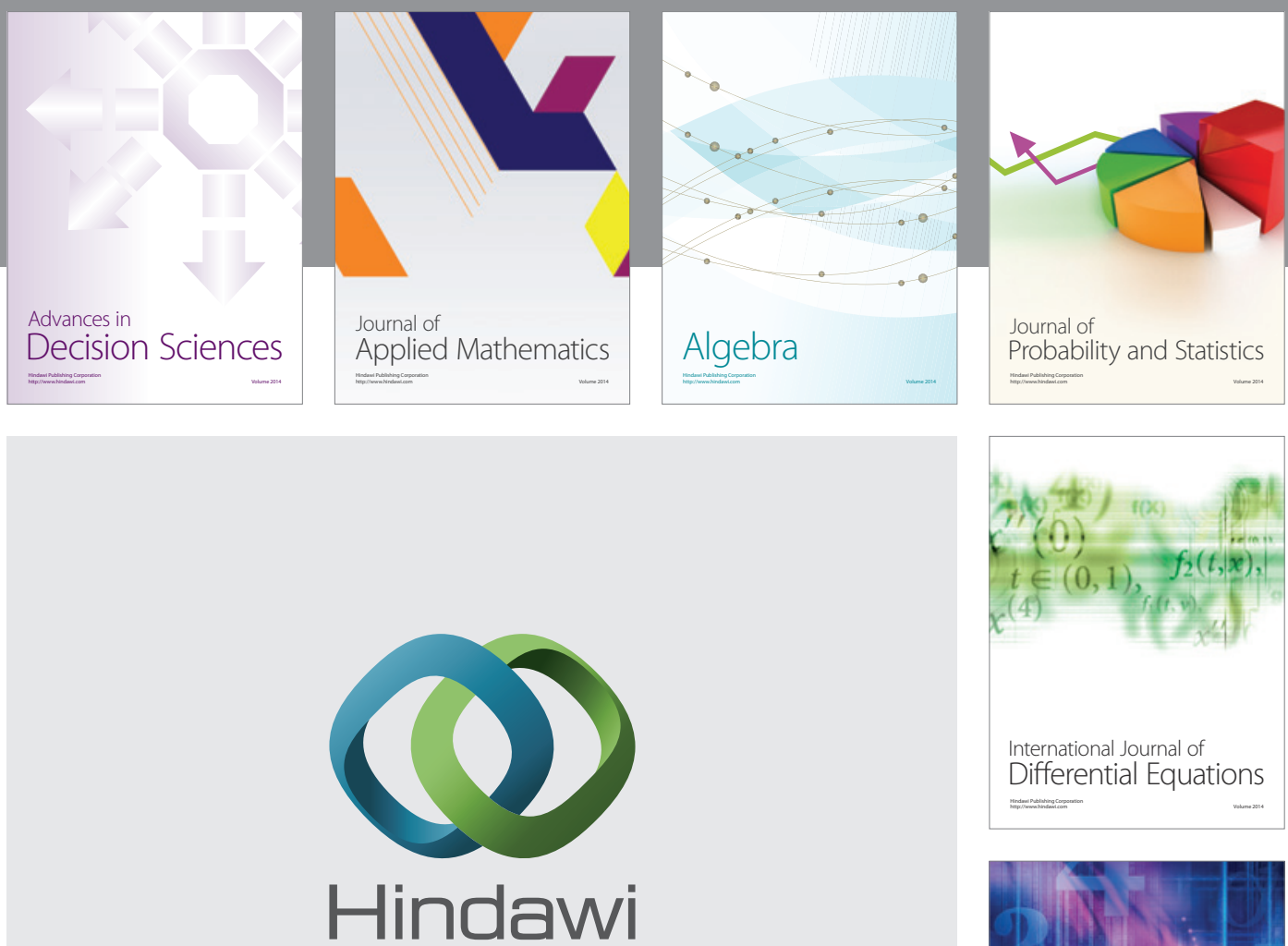

Submit your manuscripts at http://www.hindawi.com
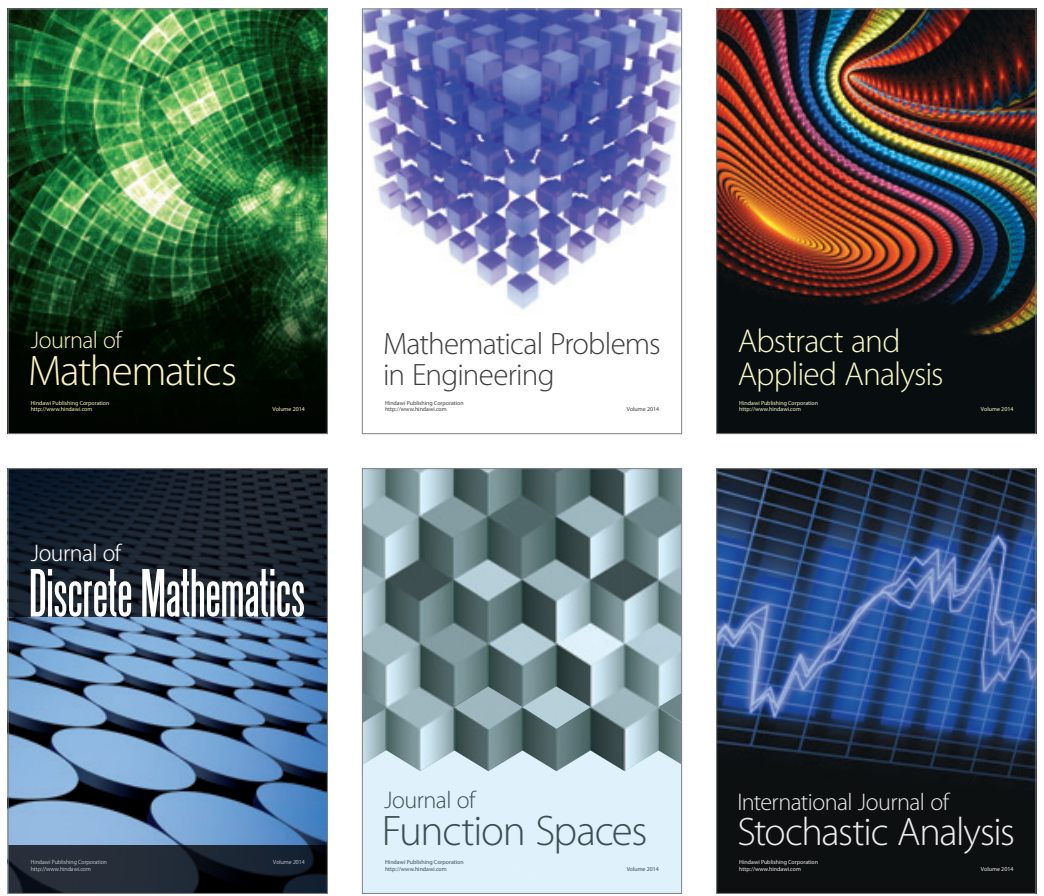

Journal of

Function Spaces

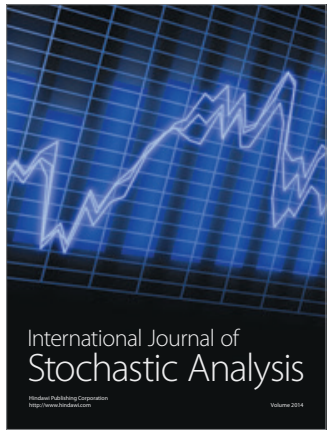

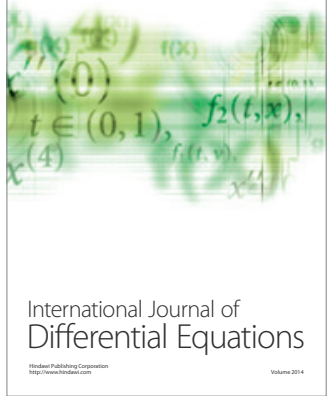
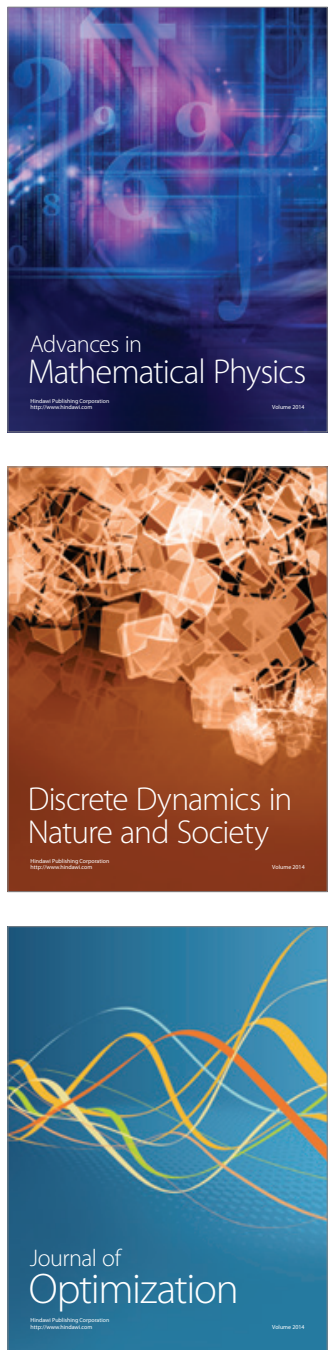